\title{
Preface for the microbial biofilm issue
}

\author{
Wen-yuan Shi ${ }^{1}$, Xue-dong Zhou $^{2}$ \\ ${ }^{1}$ School of Dentistry, Section of Oral Biology, Dental Research Service Center, University of California at Los Angeles, \\ CA 90095, USA; ${ }^{2}$ State Key Laboratory of Oral Diseases, Sichuan University, Chengdu 610041, China
}

International Journal of Oral Science (2011) 3: 47-48. doi: 10.4248/IJOS 11021

Ever since the first discovery of bacteria over 400 years ago by van Leeuwenhoek, reductionism has been used by microbiologists as they analyzed small components of individually isolated bacteria to try and understand the whole. Powered by molecular biology and genomics, modern microbiologists have realized that the whole is more than the simple sum of its parts. "System thinking" and "holism" have led microbiologists from studying individual cells to examining complex communities. Biofilm research is at the center stage of this exciting new revolution!

Biofilms are generally defined as aggregates of microorganisms that are embedded within a matrix of extracellular polymeric substances (EPS) and adhered to each other and/or to surfaces. Dr. J. William Costerton (the keynote speaker of the coming International Symposium of Microbial Biofilm in Chengdu, China) is the scientist who coined this term in 1978 when he noticed that natural bacterial colonies were creating their own microhabitat, sticking to surfaces and covering themselves with a slimy layer of protective molecules. Dr. Costerton's initial biofilm discovery illuminated an entirely new aspect of microbiological research and discoveries of biofilms spread like wild fires. Biofilms were found to be extremely common in nature. In industrial environments, biofilms can develop on the interiors of pipes and lead to clogs and corrosion. In medicine, biofilms spreading along implanted tubes or wires can lead to pernicious infections in patients. And of course, dental plaque is also a biofilm. Bacteria in a biofilm have significantly different physiological characteristics, perhaps most well known of which is increased resistance to detergents and antibiotics, making biofilm research highly important. Sponsored by West China School of Stomatology, University of California at Los Angeles, Zhejing University and Johnson \& Johnson Consumer Companies Inc., we are extremely proud to organize the first International Symposium of Microbial Biofilm in China, featuring some of the best biofilm researchers from the USA, Canada, Europe and China.

This issue of International Journal of Oral Science (IJOS) is dedicated for this conference. It includes four invited comprehensive reviews, covering topics from biofilm structures, to inter-species interactions within biofilms to controlling biofilm related diseases. It also features three invited original research articles, describing novel tools to study microbial interactions within biofilms and new findings of biofilm associated physiological and molecular changes. We trust that the information provided in these articles will be useful for you to further understand biofilm research. 
We would like to thank the West China School of Stomatology biofilm conference team and the IJOS editorial team for their hard work in preparation of this special conference and IJOS issue. We also wish to thank all sponsors for their generous support.

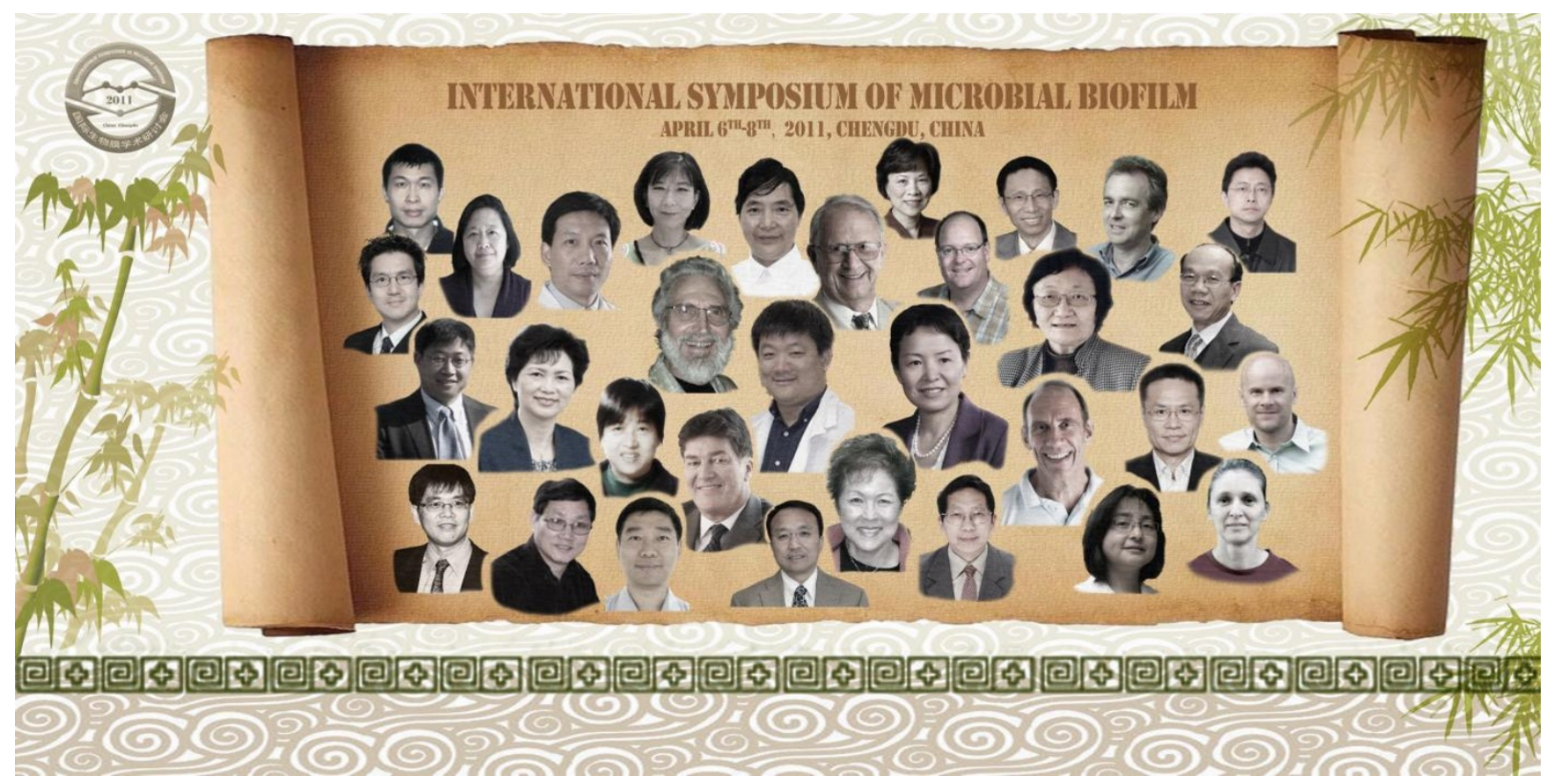

Keynote Speakers in the International Symposium of Microbial Biofilm Chengdu China April 6th-8th, 2011 